\title{
UTILIZAÇÃO DE POLÍMERO EM PILHAS DE CARVÕES E ESTUDO DE DECAIMENTO DE FLUIDEZ E MATÉRIA VOLÁTIL*
}

\author{
Guilherme Liziero Ruggio da Silva ${ }^{1}$ \\ Erick Mitchell Henrique Braga ${ }^{2}$ \\ Lorena Cristina Amorim ${ }^{3}$ \\ Leandro Rocha Lemos ${ }^{4}$ \\ Diego Silva de Deus Vieira ${ }^{5}$ \\ Verlaine Inácia de Oliveira Nóbrega ${ }^{6}$ \\ Paulo Santos Assis ${ }^{7}$
}

\section{Resumo}

As coquerias enfrentam um grande problema que é a oxidação do carvão. Este fenômeno as afeta diretamente, pois deterioram as propriedades coqueificante do material, impactando na síntese do coque metalúrgico. Portanto, gerando perda de valor econômico, uma vez que as propriedades coqueificantes são fatores que determinam a atratividade e o custo do carvão. Sendo assim, torna-se necessário o uso de medidas para retardar o efeito da oxidação, garantindo a menor perda possível, desde a extração do carvão mineral da mina, até o carregamento nos fornos de coqueificação. Neste estudo em particular, definiu-se uma equação para previsão do decaimento de fluidez, que é a principal propriedade afetada pela oxidação, e matéria volátil de carvões em pilhas a céu aberto. Foi também desenvolvido o estudo do efeito da aspersão de uma camada polimérica para minimizar os decaimentos de fluidez e matéria volátil.

Palavras-chave: Pilhas de carvão; Oxidação do carvão; Fluidez do Carvão; Impermeabilização do carvão.

\section{USE OF POLYMER ON STOCKPILE COALS AND PREDICTIONS OF FLUIDITY AND VOLATILE MATTER DECAYS}

\section{Abstract}

Coke plants face a significant problem that is coal oxidation. This phenomenon affects directly their production, because it damages the coals coking properties, affecting the metallurgical coke synthesis. Therefore, it results in economic loss, since the coking properties are critical factors to determine the attractiveness and price of coal. Thus, measures to retard the effect of oxidation are used in order to ensure the lowest possible loss, from the extraction of the mineral coal, out the mine, to the loading in the coking furnaces. In this particular study, it was defined an equation to predict the decay of fluidity, which is the main property affected by oxidation, and volatile matter in open-air coal piles. In addition, it was studied the effect of polymer sprinkling to minimize fluidity and matter volatile decays.

Keywords: Fluidity decay; Coal oxidation; Polymer Sprinkling; Coal stockpiles.

\footnotetext{
${ }^{1}$ Doutor em Engenharia dos Materiais REDEMAT/UFOP, Gerdau Ouro Branco, Brasil.

${ }^{2}$ Mestre em Engenharia dos Materiais REDEMAT/UFOP, Gerdau Ouro Branco, Brasil.

${ }^{3,6}$ Graduanda em Engenharia Metalúrgica, IFMG Ouro Branco, MG, Brasil

${ }^{4}$ Prof. Dr. Adjunto, DEMET, UFMG, Belo Horizonte, MG, Brasil

${ }^{5}$ Graduando em Engenharia Metalúrgica, DEMET, UFMG, Belo Horizonte, MG, Brasil

7 Prof. Dr. Titular da Escola de Minas da UFOP - REDEMAT, Ouro Preto, MG, Brasil
} 


\section{INTRODUÇÃO}

Carvões de todos os ranks, por serem um material carbonoso, estão sujeitos a sofrerem oxidação em baixas temperaturas, com a presença de ar rico em oxigênio [1, 2]. A interação de carvão com o oxigênio a baixas temperaturas é exotérmica. No entanto, algumas sequências de reações podem ser endotérmicas [2].

A oxidação do carvão ocorre basicamente para os macerais do grupo da vitrinita. $O$ processo consiste em reações entre o oxigênio da atmosfera e as partículas de carvão, causando uma deterioração das propriedades plásticas do mesmo. Alguns dos fatores que influenciam a oxidação do carvão são o rank, granulometria, temperatura, umidade, pressão parcial de $\mathrm{O}_{2}$, etc. Sendo a granulometria um dos parâmetros mais importantes, pois aumenta a área superficial do carvão, acelerando as reações de oxidação [3].

A oxidação da matéria orgânica do carvão é um fenômeno muito complexo já que o carvão é fisicamente heterogêneo e também uma substância química complexa. Este processo pode ser descrito como uma reação gás-sólido com a oxidação se dirigindo do exterior para o centro da partícula [4].

Ao absorver ou perder umidade a partícula de carvão tende a apresentar trincas e se degradar, pois a dilatação volumétrica da superfície sempre será mais rápida que a do interior da partícula. Este fenômeno aumenta substancialmente a área de contato da superfície do carvão com o ar [5].

Além de causar a degradação da partícula, a absorção de umidade também gera uma certa quantidade de calor. Este calor, em certos casos, pode ser até maior que $22 \mathrm{cal} / \mathrm{g}$. Portanto, se não for dissipado rapidamente, a temperatura do carvão irá aumentar e acelerar a sua oxidação [5].

O "slacking index" é um índice que simula a degradação do carvão em condições cíclicas de secagem e molhagem, sendo um teste desenvolvido pelo United States Burial of Mines, que consiste em secar uma amostra com granulometria acima de 1,05", sob uma temperatura entre 30 a $35^{\circ} \mathrm{C}$, por 24 horas, com imersão da amostra em água por uma hora e posterior secagem por mais 24 horas. Após este procedimento, mede-se o percentual em peso da amostra passante em uma peneira de 0,263 " para a obtenção deste índice [6,7]. Até $10 \%$ de umidade, não há uma taxa tão grande de degradação no carvão, a partir desse nível, essa taxa aumenta consideravelmente como podemos ver pela Figura 1.

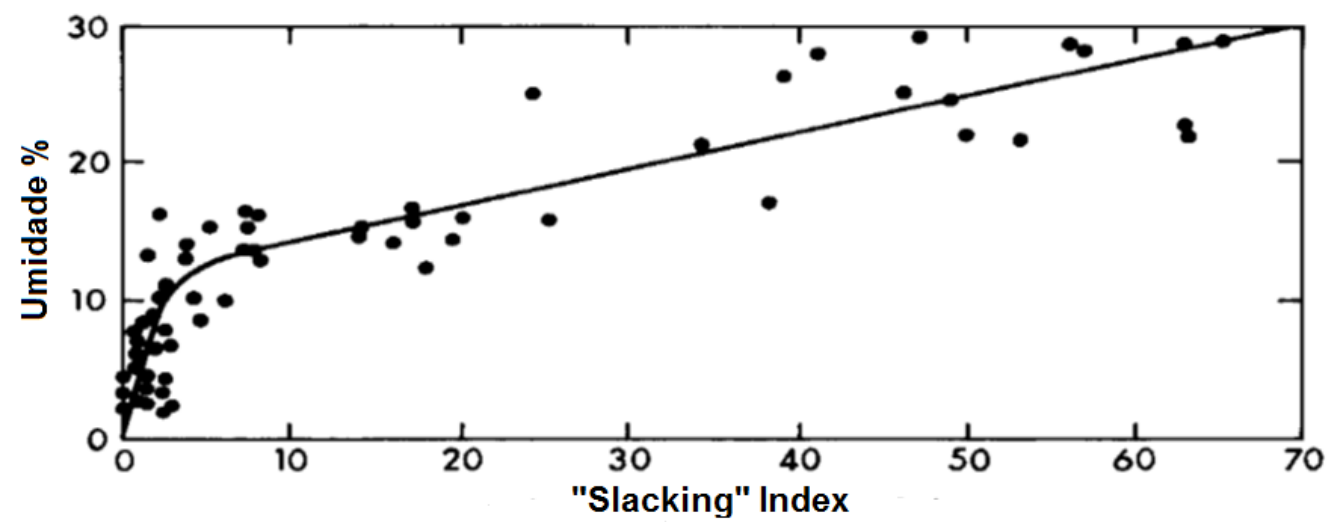

Figura 1. Variação da taxa de degradação do carvão pela umidade (adaptação de [8]). 
A taxa de oxidação depende da temperatura, geralmente dobrando a cada aumento de $10^{\circ} \mathrm{C}$, além da própria oxidação ser uma reação exotérmica (cerca de $7800 \mathrm{kcal} / \mathrm{kg}$ de carvão oxidado para $\mathrm{CO}_{2}$ ). O acúmulo desse calor na pilha de carvão irá aumentar ainda mais a taxa de oxidação, causando um aquecimento autógeno, podendo culminar na ignição espontânea do carvão [5].

Sendo assim, a ignição espontânea ocorre quando o calor que é produzido pelas reações à baixas temperaturas do carvão com o oxigênio, não é adequadamente dissipado para o ambiente. Ao atingir a temperatura de ignição, algumas chamas surgem na pilha de carvão e parte do carvão é queimado. Isto, não só introduz um custo indesejado, mas também coloca em risco a segurança do ambiente de trabalho. [9]

Sendo a oxidação uma reação de superfície, sua velocidade irá depender da taxa em que o oxigênio pode se difundir nas partículas de carvão. O que está bem correlacionado pela área superficial do carvão na qual o oxigênio tem acesso [5].

Um aumento de $1 \%$ no percentual de oxigênio pode ser suficiente para acabar com as propriedades coqueificantes dos carvões metalúrgicos [5]. Assim, um bom indicador para se verificar a oxidação do carvão é pela análise de fluidez Gieseler ou FSI [10] representada na Figura 2.

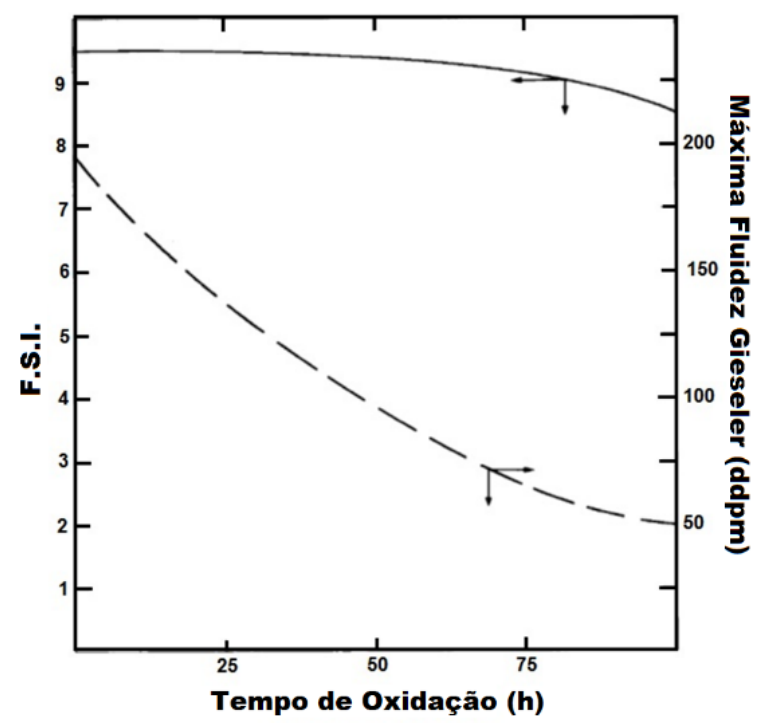

Figura 2. Variação de FSI e máxima fluidez Gieseler pelo tempo de oxidação no ar a 70ํำ (adaptação de [5]).

A fluidez mensura o quão fluido o carvão se torna quando é aquecido à temperatura de pirólise [11], ou seja, sua capacidade de se expandir e promover contato com outras partículas de carvão para a posterior ressolidificação em coque. A perda dessa importante característica impacta diretamente nas propriedades coqueificantes dos carvões metalúrgicos [12].

Durante o estágio plástico, entre 350 e $500^{\circ} \mathrm{C}$, a matéria volátil do carvão exerce pressão sobre as paredes da partícula, o que provoca a dilatação simultaneamente ao desprendimento de voláteis, conforme a Figura 3 ilustra. $\mathrm{O}$ poder coqueificante da mistura de carvões é traduzido pela capacidade de fluidificação da massa carbonosa, gerando pontos de contato entre as partículas que são responsáveis pela aglomeração metalúrgica do carvão, vital para a produção do coque. 


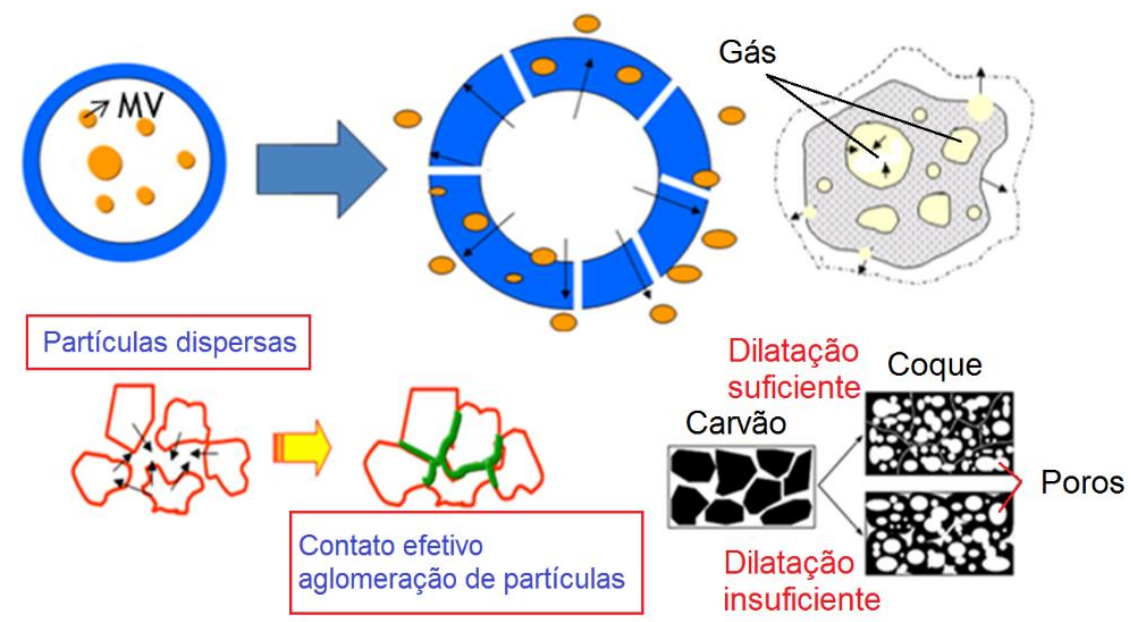

Figura 3. Efeito da dilatação do carvão sobre o coque (adaptação de [13]).

De modo geral, a taxa da queda de fluidez é proporcional à fluidez instantânea do carvão e pode ser descrita por uma equação diferencial de $1^{\text {a }}$ ordem, cuja solução evidencia o caráter exponencial da diminuição do poder coqueificante do material. A Figura 4 ilustra o exposto.

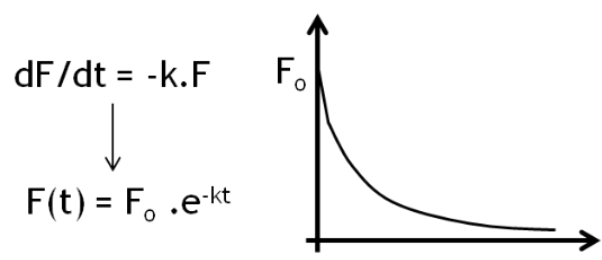

Figura 4. Degradação de fluidez do carvão.

O teste de fluidez ou plastometria fornece um comportamento muito interessante, tendo em vista que, como apresentado na Figura 5, existe um tamanho ótimo de grão que maximiza o comportamento plástico do carvão.

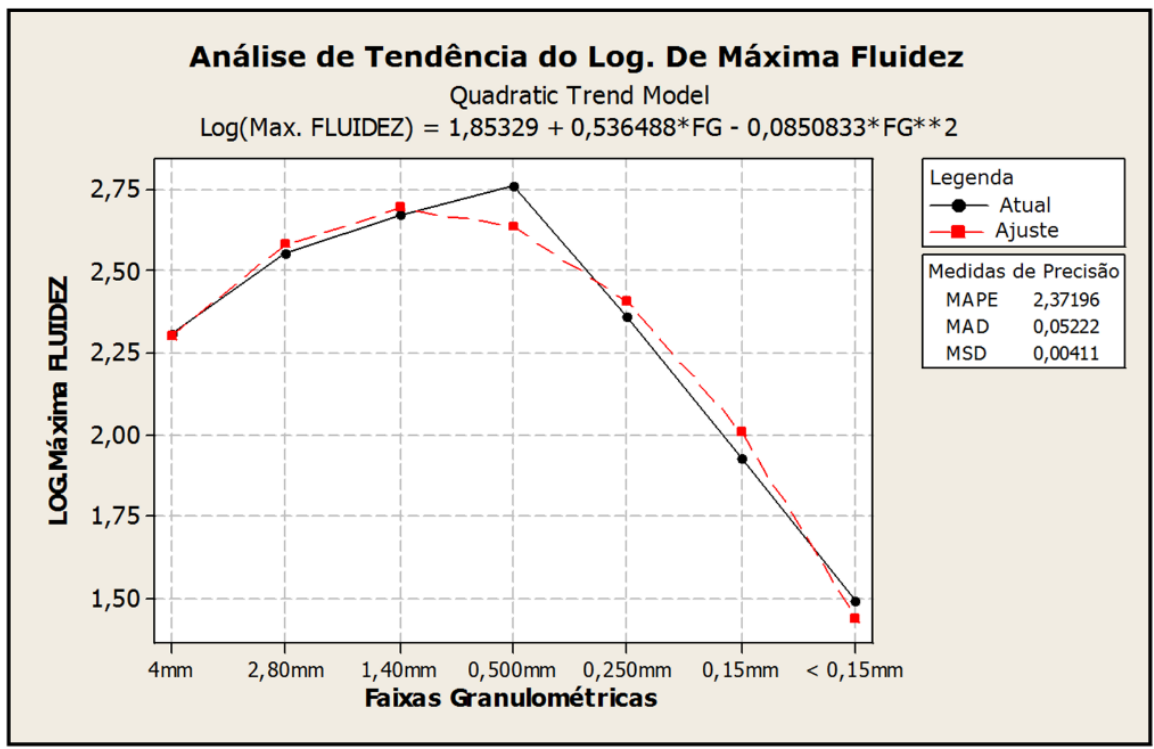

Figura 5. Relação entre tamanho da partícula de carvão e fluidez (adaptação de [14]). 
A pressão que é desenvolvida no interior do grão de constituintes reativos pela formação de poros, provoca o seu inchamento, o que é um importante fator na formação de coque. Ulhôa [15] confirmou a existência, para o tamanho de grão em função da formação de poros de:

- Um limite superior ligado à possibilidade de formação de um número excessivo de poros e, em consequência, um inchamento excessivo responsável pela formação de coque esponja;

- Um limite inferior, em torno de $0,2 \mathrm{~mm}$ a partir do qual não há mais formação de poros, o que influenciará negativamente na fluidez e, consequentemente, na resistência do coque. Assim, em termos de fluidez, o ideal é um tamanho de grão por volta de $0,5 \mathrm{~mm}$.

Através do teste de plastometria são obtidas as seguintes temperaturas: de início de dilatação, de amolecimento, de máxima fluidez, de fim de dilatação e de início de solidificação. Com base nos valores das temperaturas de amolecimento e de início de solidificação é obtido o chamado range plástico dado pela diferença entre estas temperaturas.

A correlação entre range plástico e granulometria está apresentada na Figura 6. Com base neste índice, pode-se concluir que o tamanho de grão ideal está compreendido no intervalo $[0,2 ; 0,5] \mathrm{mm}$. Outro aspecto interessante é a queda brusca no range plástico para o material abaixo de 0,15 $\mathrm{mm}$ (superfino), o que contribui para a conjectura de que este material, em excesso, é prejudicial à coqueificação.

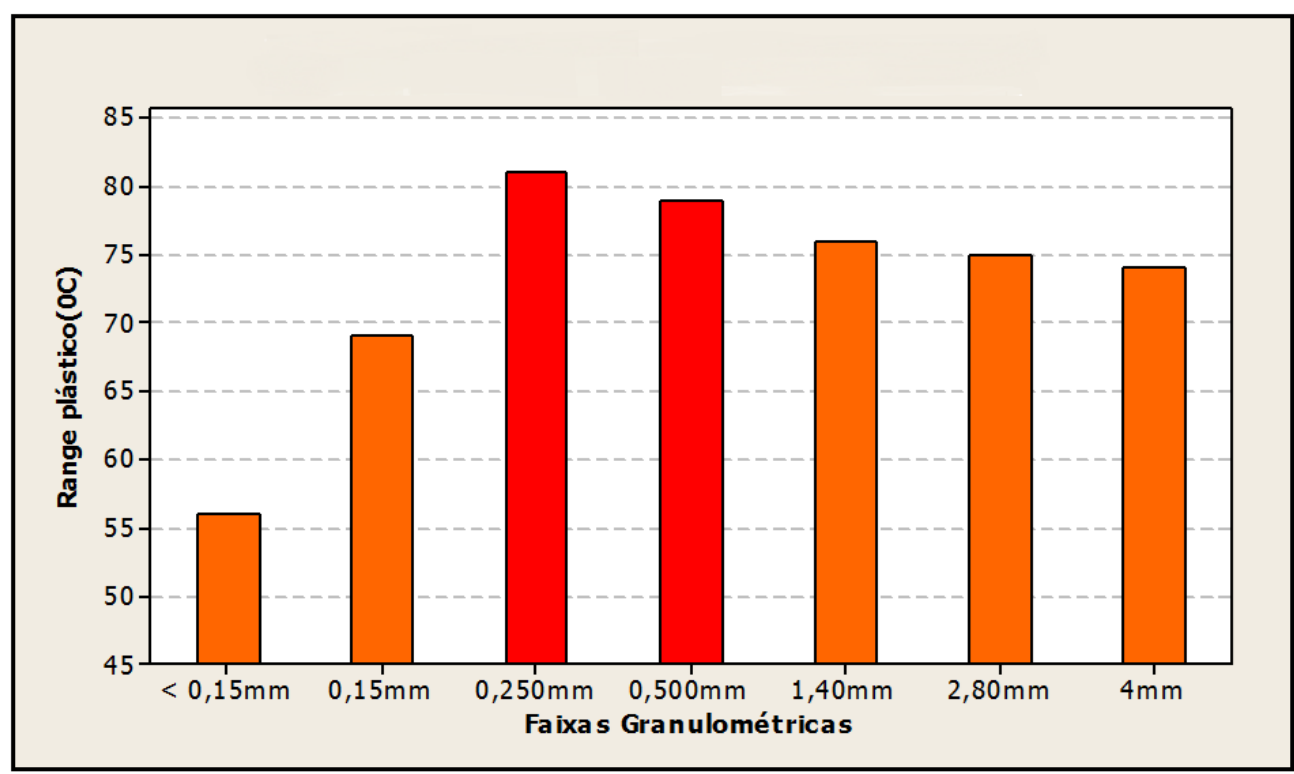

Figura 6. Perfil do range plástico versus faixa granulométrica.

Os coques produzidos a partir de carvões oxidados tendem a possuir maior reatividade (CRI) e menor resistência mecânica a quente e a frio (CSR e DI) [3].

Para prevenir a oxidação no carvão, algumas medidas podem ser tomadas, como por exemplo: as temperaturas durante a estocagem serem as menores possíveis (acima de $0^{\circ} \mathrm{C}$ ); a estocagem do carvão ser realizada em atmosfera com baixa concentração ou livre de oxigênio, ou sob a água; antioxidantes que podem ser adicionados ao carvão para retardar a oxidação por alguns meses [5]; impermeabilização das pilhas para reduzir a interface do carvão com a atmosfera por meio de agentes inibidores [17]. 
O uso de inibidores tem sido vastamente utilizado em minas de carvão, com o intuito de prevenir o aquecimento do carvão e consequentemente, incêndios. Sendo que existem dois grandes grupos de inibidores, os físicos e os químicos. [16-18]

Os inibidores físicos são capazes de absorver água e prevenir o oxigênio de atingir o centro do carvão por meio de difusão. Sendo, os principais exemplos desse tipo de inibidores sais hidroscópicos (como $\mathrm{NaCl}, \mathrm{MgCl}_{2}$ e $\mathrm{CaCl}_{2}$ ), espuma trifásica, vidro de água (composto de silicato de sódio $\mathrm{Na}_{2} \mathrm{O}_{3} \mathrm{Si}$ ). Estes agentes são largamente utilizados em minas de carvão, no entanto, eles apresentam baixa eficiência e são rapidamente consumidos. [18]

Já o grupo dos inibidores químicos é representado por agentes oxidantes, soluções iônicas e sais inorgânicos (como por exemplo $\mathrm{Na}_{3} \mathrm{PO}_{4}$ e $\left.\mathrm{Mg}(\mathrm{Ac})_{2}\right)$. Este tipo de agentes inibidores são capazes de reagirem com os grupos funcionais ativos presentes na superfície das partículas de carvão, resultando na redução da formação de grupos ativos ou diminuindo sua atividade durante as reações com o oxigênio. Estas substâncias são altamente eficientes, porém alguns desses inibidores apresentam elevado preço ou a desvantagem de não serem aplicáveis a certos tipos de carvões. [16]

Um terceiro grupo de inibidores seriam os inibidores de base polimérica. Este grupo funciona a partir da formação de uma fina membrana sobre a superfície do carvão. Sendo assim, prevenindo reações entre as partículas de carvão com o oxigênio. [18]

Neste trabalho será abordada a técnica de aspersão de camada polimérica sobre pilhas de carvão, para evitar emissão de particulados. Técnica que também pode ser eficaz contra a oxidação, funcionando como um agente inibidor de base polimérica.

\section{MATERIAIS E MÉTODOS}

O presente trabalho foi dividido em duas partes práticas, uma utilizando carvão médio volátil (MRA) e outra, carvão alto volátil (AWL). Para o carvão MRA foram realizadas análises de matéria volátil e fluidez, no intuito de determinar uma equação para decaimento de fluidez. Já para o carvão AWL, foram preparadas amostras em escala piloto para a análise de fluidez, para determinar a eficiência da camada de polímero em retardar a oxidação do carvão.

Em ambas as partes, foi necessária a realização do teste de fluidez, conhecido como plastometria. O teste consiste em um cadinho com $5 \mathrm{~g}$ de carvão < 35 mesh, aquecido em ausência de ar. Um pequeno agitador, posicionado no seio da massa de carvão e submetido a um torque constante, é acoplado a um motor de 300 rpm e a um dial com 100 divisões (leituras de até $30.000 \mathrm{ddpm}$ ). O torque é tal que 0 agitador não possa girar quando o carvão ainda está sólido. A amostra-teste é aquecida numa taxa de $3^{\circ} \mathrm{C} / \mathrm{min}$ entre $300^{\circ} \mathrm{C}$ e $500^{\circ} \mathrm{C}$.

\subsection{Parte 1 - Teste MRA (escala industrial)}

Primeiramente, para avaliar o decaimento de fluidez e de matéria volátil, uma pilha de carvão médio volátil (MRA), com cerca de 4000t e propriedades descritas na Tabela 1, foi estocada por 900 dias num pátio a céu aberto, sofrendo ação natural de intempéries e mudanças climáticas, antes de ser consumida. Nesse período, várias amostras desta pilha foram recolhidas e caracterizadas, a fim de determinar a ação do tempo e método de estocagem nas propriedades coqueificantes do carvão. 


\subsection{Parte 2 - Teste AWL (escala piloto)}

Um segundo teste foi realizado com a intenção de criar um filme protetor sobre as pilhas de carvão mineral. Este filme seria responsável por impermeabilizar as pilhas da ação do oxigênio e umidade, como mostrado na Figura 7. Para este experimento foram utilizadas amostras de carvão alto volátil (AWL) com as propriedades descritas na Tabela 1 abaixo.

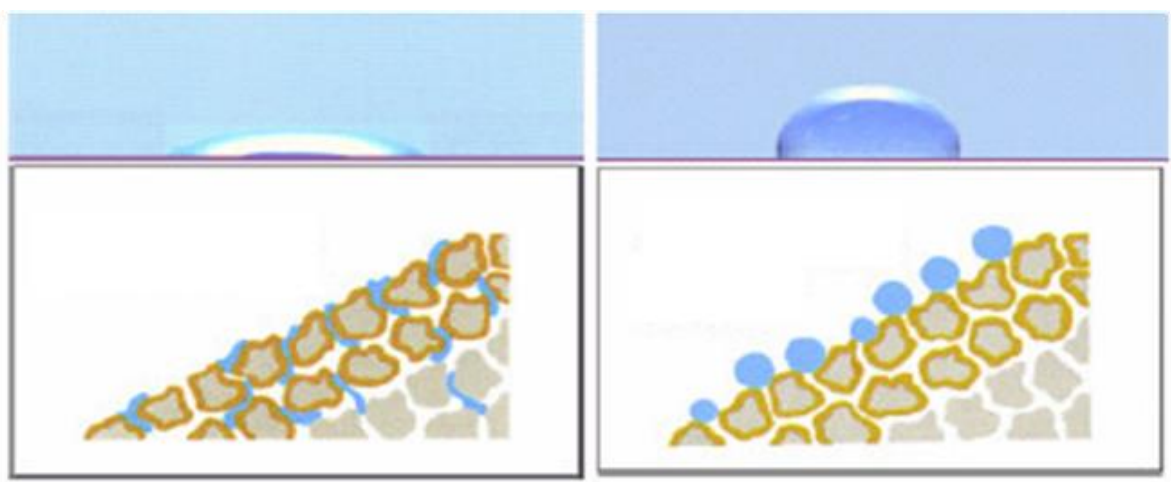

Figura 7. Desenho esquemático com impermeabilização da camada externa de carvão.

Tabela 1. Propriedades iniciais do MRA e AWL

\begin{tabular}{|c|c|c|c|c|c|c|c|c|}
\hline \multirow[b]{2}{*}{ Carvão } & \multicolumn{2}{|c|}{ Máxima Fluidez } & \multirow{2}{*}{$\begin{array}{l}\mathrm{TA}^{*} \\
\left({ }^{\circ} \mathrm{C}\right)\end{array}$} & \multirow{2}{*}{$\begin{array}{l}\text { TMF }^{*} \\
\left({ }^{\circ} \mathrm{C}\right)\end{array}$} & \multirow{2}{*}{$\begin{array}{l}\mathrm{TR}^{*} \\
\left({ }^{\circ} \mathrm{C}\right)\end{array}$} & \multirow{2}{*}{$\begin{array}{c}\text { Cinza } \\
(\%)\end{array}$} & \multirow{2}{*}{$\begin{array}{l}\text { MV* }^{*} \\
(\%)\end{array}$} & \multirow{2}{*}{$\begin{array}{c}\text { Enxofre } \\
(\%)\end{array}$} \\
\hline & (ddpm) & $\begin{array}{l}\text { Logaritmo } \\
\text { (LOGddpm) }\end{array}$ & & & & & & \\
\hline AWL & 10070 & 4,003 & 379 & 430 & 463 & 6,4 & 33,66 & 0,922 \\
\hline
\end{tabular}

Sendo assim, foram preparadas 6 bandejas contendo, cada uma, uma pilha com $500 \mathrm{~g}$ de carvão de alta fluidez. Em 3 dessas pilhas foi aspergida uma solução de água com 3\% em massa do polímero Kurita GF-1910, a uma razão de $100 \mathrm{~g}$ do polímero/ $\mathrm{m}^{2}$ de superfície exposta, com o objetivo de formar um filme durável sobre a superfície das pilhas de materiais, sendo um agente supressor de pó e estabilizador da pilha.

Para acelerar os efeitos da oxidação, as pilhas foram acondicionadas em uma mufla com temperatura constante de $35^{\circ} \mathrm{C}$ durante 8horas/dia, (9-16h) e o restante do tempo, à temperatura ambiente. Foram retiradas amostras a cada quatro semanas para avaliar a propriedades do carvão com e sem a camada polimérica.

\section{RESULTADOS E DISCUSSÃO}

\subsection{Parte 1 - Teste MRA}

Os resultados das análises da pilha de MRA durante o tempo de estocagem estão mostrados na Tabela 2. 
Tabela 2. Análises do carvão MRA no tempo

\begin{tabular}{cccccccc}
\hline $\begin{array}{c}\text { Tempo de } \\
\text { estocagem } \\
\text { (dias) }\end{array}$ & MV $^{*}(\%)$ & $\begin{array}{c}\text { Máx } \\
\text { Flu } \\
\text { (ddpm) }\end{array}$ & $\begin{array}{c}\text { Log. Máx } \\
\text { Fluidez } \\
(\text { LOGddpm) }\end{array}$ & $\begin{array}{c}\text { TA }^{*} \\
\left({ }^{\circ} \mathbf{C}\right)\end{array}$ & $\begin{array}{c}\text { TMF }^{*} \\
\left({ }^{\circ} \mathbf{C}\right)\end{array}$ & $\begin{array}{c}\text { TR }^{*} \\
\left({ }^{\circ} \mathbf{C}\right)\end{array}$ & $\begin{array}{c}\text { Range } \\
\text { Plástico }\end{array}$ \\
\hline $\mathbf{0}$ & 31,08 & 3987 & 3,60 & 386 & 426 & 467 & 81 \\
$\mathbf{3 6}$ & 30,81 & 9532 & 3,98 & 380 & 448 & 493 & 113 \\
$\mathbf{6 2}$ & 28,37 & 638 & 2,81 & 385 & 435 & 464 & 79 \\
$\mathbf{1 3 6}$ & 30,28 & 5249 & 3,72 & 393 & 450 & 489 & 96 \\
$\mathbf{2 6 9}$ & 28,66 & 190 & 2,28 & 414 & 452 & 486 & 72 \\
$\mathbf{2 6 9}$ & 28,83 & 230 & 2,36 & 388 & 432 & 459 & 71 \\
$\mathbf{3 3 0}$ & 30,21 & 11 & 1,04 & 399 & 436 & 448 & 49 \\
$\mathbf{3 7 1}$ & 30,56 & 233 & 2,37 & 401 & 442 & 467 & 66 \\
$\mathbf{8 5 4}$ & 28,20 & 25 & 1,40 & 400 & 439 & 474 & 74
\end{tabular}

*TA: Temperatura de Amolecimento; ${ }^{*} T M F$ : Temperatura de Máxima Fluidez; ${ }^{*} T R$ : Temperatura de Ressolidificação; *MV: Matéria Volátil;

O decaimento do logaritmo de máxima fluidez, se comporta como na Figura 8 em função da quantidade de dias que o material é estocado. Com base nesses dados, obteve-se a equação 1 que prevê a máxima fluidez.

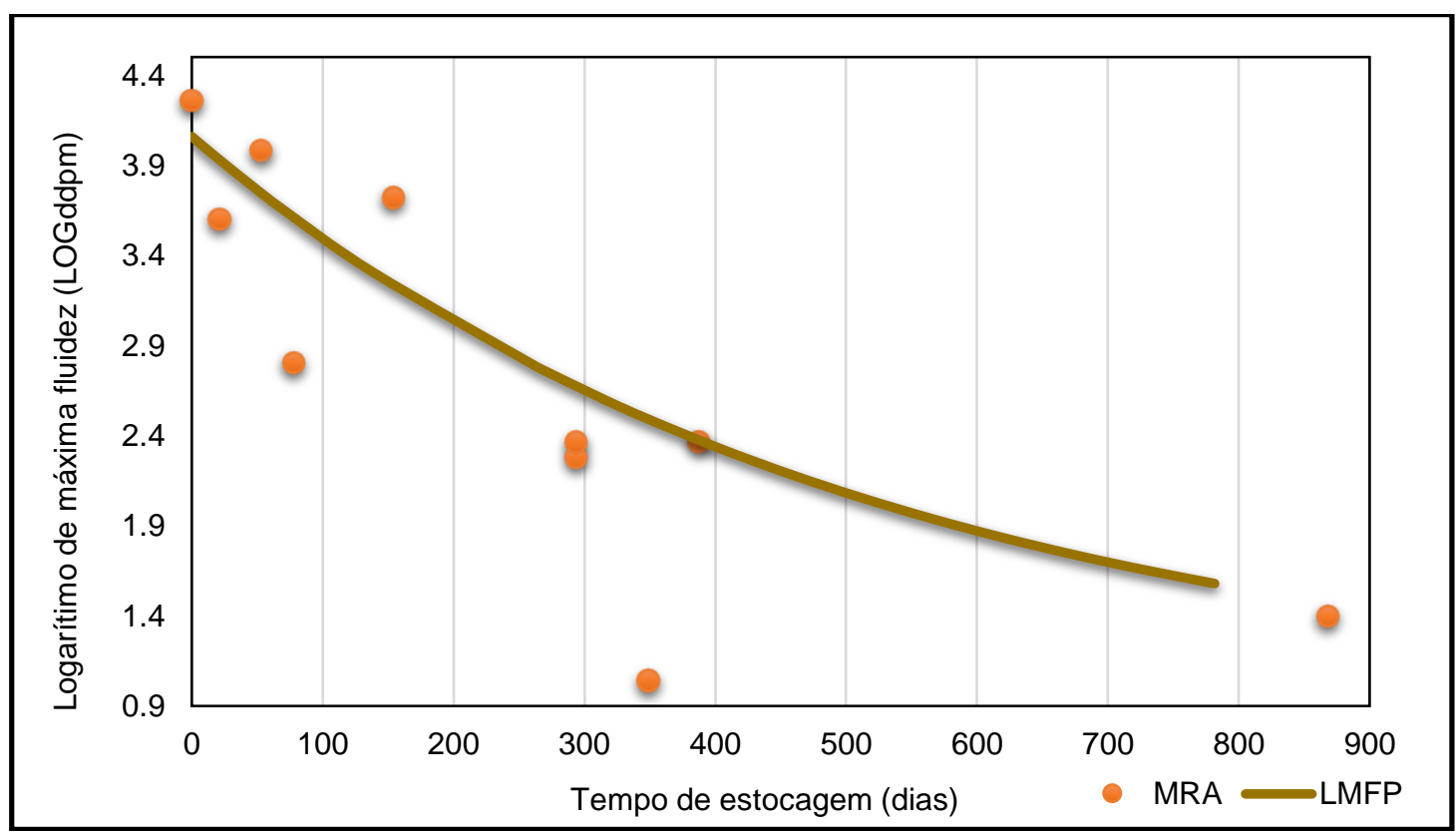

Figura 8. Decaimento do logarítimo de máxima fluidez com o tempo de estocagem.

$$
\mathbf{L M F}=3,97448 \times \operatorname{EXP}\left(-0,00176927 \times\left(\mathbf{L M F I}+\frac{\operatorname{Ln}\left(\frac{\mathrm{DEE}}{3,97448}\right)}{-0,00176927}\right)\right)
$$

Onde LMFP (Logaritmo de máxima fluidez previsto) é o logaritmo de máxima fluidez calculado/previsto; LMFI (Logaritmo de máxima fluidez inicial) é o logaritmo de máxima fluidez no momento da formação da pilha; DEE (Dias em estoque) é o tempo em dias que a pilha está estocada.

Para determinar o decaimento da matéria volátil com o tempo, foram combinados os dados do carvão em estudo (MRA) e os carvões estudados por Bend et al. [19] (propriedades na Tabela 3), com o MRA defasado no tempo, como demonstrado pela Figura 9. Com base nesses dados, chegamos a Equação 2. 
Tabela 3. Propriedades iniciais dos carvões estudados por Bend et al [19]

\begin{tabular}{|c|c|c|c|c|c|c|}
\hline \multirow{2}{*}{ Carvão } & \multirow{2}{*}{ Rank (ASTM) } & \multicolumn{3}{|c|}{ Macerais (\%) } & \multirow{2}{*}{$\begin{array}{c}\text { Matéria } \\
\text { Volátil (\%) }\end{array}$} & \multirow{2}{*}{ Cinzas (\%) } \\
\hline & & Vitrinita & Inertinita & Matéria Mineral & & \\
\hline $88 / 025$ & $\mathrm{Hvb} B$ & 61,2 & 23,8 & 0,6 & 39 & 4,8 \\
\hline $88 / 026$ & $\mathrm{Hvb} A / B$ & 76,4 & 11,6 & 0 & 35 & 10,02 \\
\hline $88 / 024$ & Hvb A & 72,2 & 14,8 & 0,6 & 37 & 5,1 \\
\hline
\end{tabular}

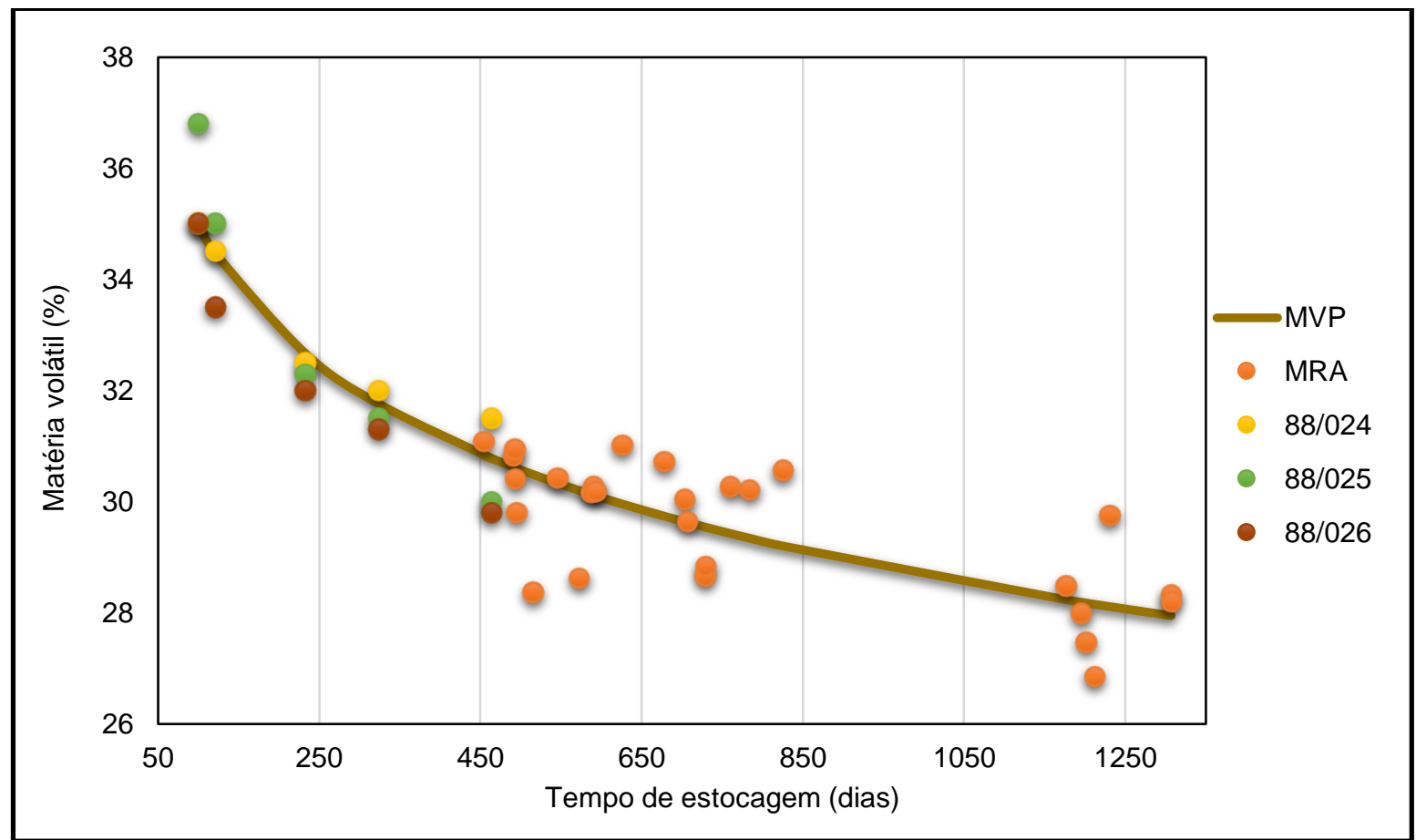

Figura 9. Decaimento de matéria volátil com o tempo de estocagem

$\mathbf{M V P}=-2,72363 \times \operatorname{Ln}\left(\mathbf{D E E}+100+\operatorname{Exp}\left(\left(\frac{\mathbf{M V I}-47,488}{-2,72363}\right)\right)-100\right)+47,488$

Onde MVP (Matéria volátil prevista) é o percentual de matéria volátil calculado/previsto; MVI (Matéria volátil inicial) é o percentual de matéria volátil no momento da formação da pilha; DEE (Dias em estoque) é o tempo em dias que a pilha está estocada.

\subsection{Parte 2 - Teste AWL}

A Tabela 4 apresenta os resultados de fluidez das amostras. Já, a diferença entre a máxima fluidez (ddpm) das amostras com e sem adição de polímero, pode ser observada na Figura 10. 
Tabela 4. Propriedades coqueificantes das amostras submetidas à oxidação à $35^{\circ} \mathrm{C}$.

\begin{tabular}{|c|c|c|c|c|c|c|c|}
\hline & \multirow{2}{*}{$\begin{array}{l}\text { Amostra } \\
\text { Inicial }\end{array}$} & \multicolumn{6}{|c|}{ Bandejas } \\
\hline & & 1 & 2 & 3 & 4 & 5 & 6 \\
\hline $\begin{array}{l}\text { Aplicação de } \\
\text { Polímero }\end{array}$ & Não & Não & $\operatorname{Sim}$ & Não & Sim & Não & Sim \\
\hline Fluidez (ddpm) & 10070 & 8472 & 9897 & 2500 & 4623 & 491 & 1017 \\
\hline $\begin{array}{c}\text { Fluidez } \\
\text { (LOGddpm) }\end{array}$ & 4,003 & 3,928 & 3,996 & 3,398 & 3,665 & 2,691 & 3,007 \\
\hline Diferença & 0 & 1598 & 173 & 7570 & 5447 & 9579 & 9053 \\
\hline Data & $27 / 06 / 2016$ & \multicolumn{2}{|c|}{$25 / 07 / 2016$} & \multicolumn{2}{|c|}{$22 / 08 / 2016$} & \multicolumn{2}{|c|}{$20 / 10 / 2016$} \\
\hline Dias & 0 & \multicolumn{2}{|c|}{28} & \multicolumn{2}{|c|}{56} & \multicolumn{2}{|c|}{115} \\
\hline
\end{tabular}

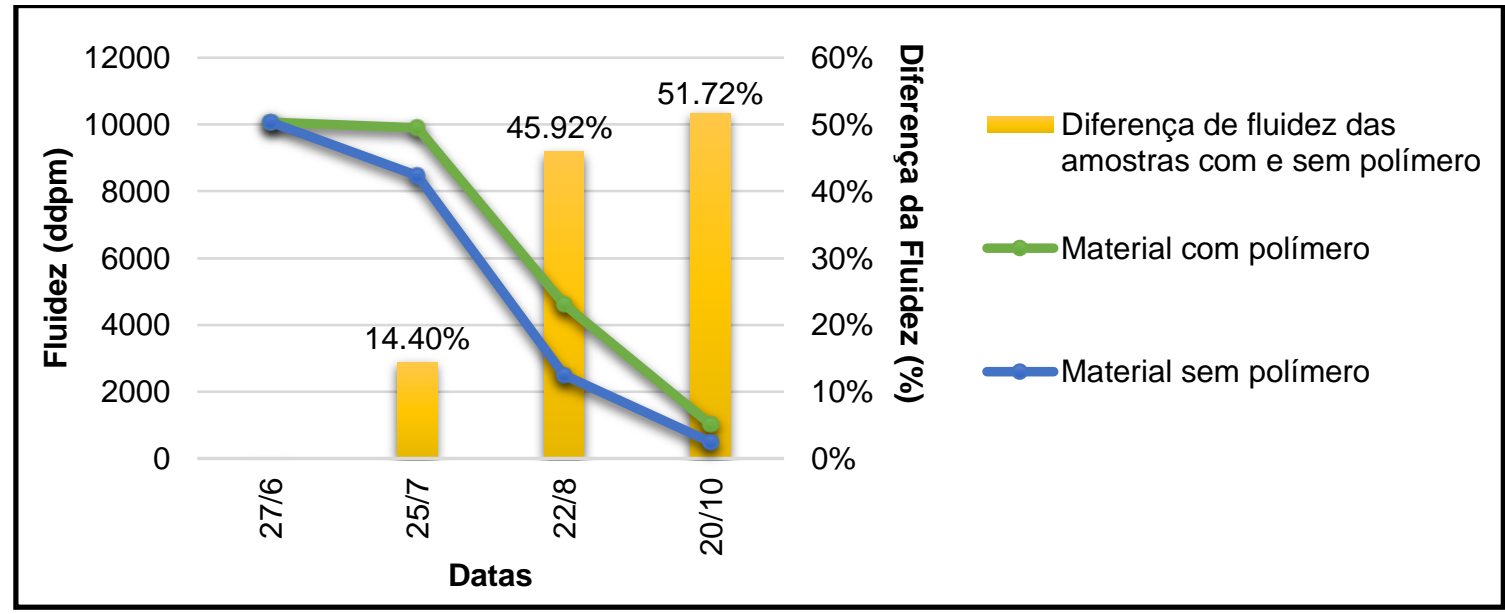

Figura 10. Efeito supressor do polímero no decaimento de fluidez.

\section{CONCLUSÃo}

Com base nas equações propostas, os carvões com baixa fluidez e matéria volátil tendem a não sentirem um efeito de oxidação pronunciado, sendo que os carvões que apresentam altos valores nestes dois índices são mais susceptíveis a redução de seu poder coqueificante.

Com posse de uma previsão de perda de propriedades coqueificantes em relação ao tempo, é possível melhorar a assertividade de previsão da qualidade de misturas de carvão e determinar logística e tempo máximo de estocagem para cada tipo de carvão.

A técnica de aspergir polímero em pilhas de carvões, além de reduzir emissão de particulados e melhorar a estabilidade da pilha, minimiza o efeito da oxidação. Portanto, esta é uma alternativa a ser considerada para a preservação de propriedades coqueificantes, principalmente dos carvões de alta volatilidade, na estocagem em pátios de carvão.

\section{REFERÊNCIAS}


1 Kozinc J, Zupancic-Kralj L, Zapusek A. Evaluation of gas emissions from coal stockpile. Chemosphere. 2003; 55: 1121-1126.

2 Zhang J, Ren T, Liang Y, Wang Z. A review on numerical solutions to self-heating of coal stockpile: Mechanism, theoretical basis, and variable study. Fuel. 2016; 182: 80109.

3 Flores IV. Avaliação da influência do tamanho de partícula sobre as propriedades de carvões coqueificáveis e de coques produzidos em escala de laboratório [dissertação para obtenção do título de mestre] Porto Alegre: Universidade Federal do Rio Grande do Sul; 2014.

4 De Korte GJ. Beneficiation of Weathered Coal. COALTECH 2020. Division of Mining Technology CSIR. 2001 [acesso em 04 abr. 2017] Disponível em:

http://www.coaltech.co.za/chamber\%20databases\%5Ccoaltech\%5CCom_DocMan.ns f/0/D1887A4A1676BCD142257436002B53EC/\$File/Oxidised\%20Coal\%20Report.pdf

5 Fryer JF, Szladow AJ. Information Series 66 - STORAGE OF COAL SAMPLES. 1973 [acesso em 04 abr. 2017]. Disponível em:

http://ags.aer.ca/document/INF/INF_066.PDF.

6 Fieldner AC, Selvig WA, Frederic WH. Accelerated laboratory test for determination of slacking characteristics of coal. Bureau of Mines, Washington, DC, USA; 1930.

7 Searight WV. Slacking properties of South Dakota coals.1932; 12. University of South Dakota.

8 Yancey HF, Johnson NJF, Selving WA. U. S. Bureau of Mines Tech. 1932; Paper 512.

9 Ejlali A, Aminossadati SM, Beamish K, Hooman BB. A new criterion to design reactive coal stockpiles. International Communications in Heat and Mass Transfer. 2009; 36: 669-673.

10 Larsen JW, Lee D, Schmidt T, Grint A. Multiple mechanisms for the loss of coking properties caused by mild air oxidation. Fuel. 1986; 65(4): 595-596.

11 Sakurovs R, Lynch LJ, Maher TP. The prediction of the fusibility of coal blends. Fuel Processing Technology. 1994; 37: 25-269.

12 Ryan B, Gransden J, Price J. Fluidity of western Canadian coals and its relationship to other coal and coke properties. 1997.

13 Kubota Y, Nomura S, Arima T, Kato K. Effects of coal inertinite size on coke strength. ISIJ International. 2008; 48(5): 563-571.

14 Silva GLR, Destro E, Marinho GM, Assis PS. Caracterização química, física e metalúrgica das frações granulométricas da mistura de carvão da Gerdau Açominas. In: III Congresso Brasileiro de Carvão Mineral; 2011; Gramado, Brasil.

15 Ulhôa MB. Caracterização e classificação de carvão para coque de alto-forno. Carvão metalúrgico para a siderurgia. 1991; Volta Redonda, Brasil.

16 Dou G, Wang D, Zhong X, Qin B. Effectiveness of catechin and poly(ethylene glycol) at inhibiting the spontaneous combustion of coal. Fuel Processing Technology. 2014; 120: $123-127$.

17 Wang D, Dou G, Zhong X, Xin H, Qin B. An experimental approach to selecting chemical inhibitors to retard the spontaneous combustion of coal. Fuel. 2014; 117: 218-223.

18 Qin B, Dou G, Wang Y, Xin H, Ma L, Wang D. A superabsorbent hydrogel-ascorbic acid composite inhibitor for the suppression of coal oxidation. Fuel. 2017; 190: 129135.

19 Bend SL, Edwards IAS, Marsh $\mathrm{H}$. The effects of oxidation and weathering on coal combustion. Abstracts of papers of the American chemical society. 1989; 198: 1155. 